\title{
Commensurability in the Solar System
}

\author{
Jan C.A. Boeyens \\ Unit for Advanced Study, University of Pretoria
}

August 13, 2009

\begin{abstract}
The features in common between the well-known Bode-Titius law, other commensurable astronomical relationships, and the organization of self-similar atomic systems, are used to demonstrate that the orbital data of all planets, asteroids, moons and rings in the solar system, reduce to a simple numerical pattern based on the golden ratio. A set of integers, not unlike the quantum numbers of atomic systems define the mean orbits of all planets and major satellites.
\end{abstract}

Résumé: Les aspects en commun entre la bien connue loi de Bode-Titius, autres relations astronomiques commensurables, et l'organisation des systmes atomiques auto-semblables, sont utiliss pour dmontrer que les donnes orbitales des plantes, astrodes, lunes et anneaux dans le systme solaire, se rduisent a un simple modle numrique bas sur le nombre dor. Un ensemble de nombres entiers, pas dissemblable aux nombres quantiques des systmes atomiques dfini les orbites moyennes de toutes plantes et satellites majeurs.

Key words: Solar system; Bode-Titius law; Planetary moons; Asteroid belt; Planetary rings; Golden ratio; Nebular theory.

\section{INTRODUCTION}

The structure of the solar system only became an issue once the geocentric model of the universe was finally abandoned by the middle of the 17th century, following the mathematical formulation of Kepler's laws. General acceptance of the heliocentric arrangement of the planets followed Laplace's 
demonstration that the entire solar system behaved as a dynamically stable Newtonian gravitational system. The ancient dream of harmony in the celestial spheres was partially restored by the discovery of minor planets in the asteroid belt, as predicted by the empirical Bode-Titius law of 1772 . This law specifies the mean radii of planetary orbits, in astronomical units of $1.496 \times 10^{8} \mathrm{~km},(\mathrm{AU})$ as:

$$
r=a+b c^{n}
$$

in which $a=0.4, b=0.3, c=2$ and $n=-\infty, 0,1,2, \ldots$ for the planets from Mercury to Uranus, including the asteroid belt, represented here by Ceres at $n=3$. The formula overestimates the orbital radius of Neptune by $25 \%$.

It has been $\operatorname{argued}^{1}$ (p. 42) that there may be a sound physical reason for this numerical regularity. Not only is there considerable evidence to suggest a morphological relationship between members of the solar system and particles in Saturn's rings, but also for commensurable mean motions of many planetary satellites. It was, for instance, shown by Kirkwood $(1866)^{1}$ (p. 262) that there are gaps, comparatively free of minor planets, at distances from the sun where their orbital periods would be simple fractions of Jupiter's period of revolution. The gaps are most noticeable at solar distances corresponding to fractional revolution periods, compared to Jupiter, of:

$$
\frac{2}{3}\left[\frac{3}{5} \frac{4}{7} \frac{5}{9} \frac{6}{11}\right] \frac{1}{2}\left[\frac{5}{11} \frac{4}{9} \frac{3}{7} \frac{2}{5}\right] \frac{1}{3}
$$

At the $\frac{2}{3}$ position an accumulation, rather than a depletion of asteroids, occurs. By comparison with a study ${ }^{2}$ of the composition of stable nuclides, expressed as rational fractions $Z / N$, representing proton:neutron ratios, the observed sequence of gaps is recognized as limiting values of Simple Farey sequences in the interval $\left(\frac{2}{3}, \frac{1}{3}\right)$, i.e $\{f\}=n /(2 n \pm 1), n=1 \rightarrow 6$. This observation is interpreted to define self-similarity between nuclide stability and the stability of both asteroid ribbons and the rings of Saturn.

In the case of nuclides the overall stability is conditioned by the convergence of $Z / N$ to the golden ratio, $\tau=0.6180 \ldots$, the well-known parameter associated with Fibonacci phyllotaxis and natural spirals, including galactic structures, exemplified here by the galaxy M51, shown in Fig. 1.

The intriguing possibility arising from this widespread self-similarity is that the regularity described by the Bode-Titius law reflects a planetary arrangement which can also be described by a sequence of rational fractions and/or a generating golden logarithmic spiral. 


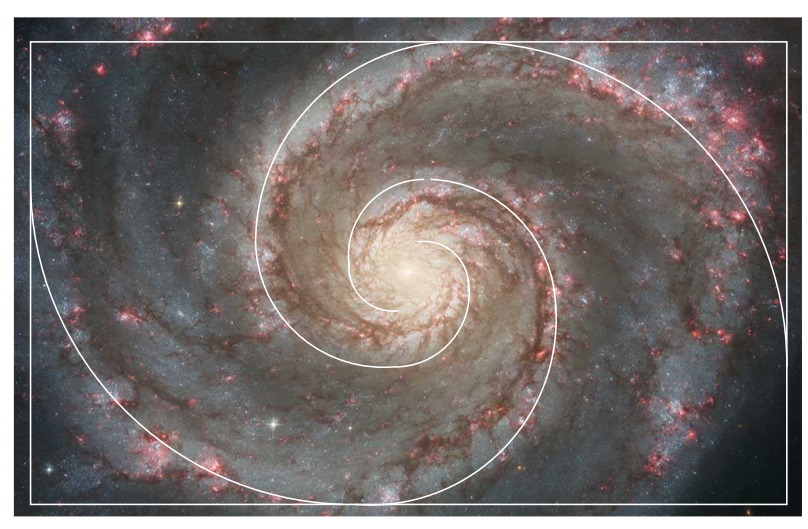

FIG. 1. (Color online) Whirlpool galaxy M51 (Courtesy Hubble Heritage Team, ESA, NASA) with a golden spiral superimposed.

\section{PLANETARY DATA}

The mean sizes of the planetary orbits including a mean path for the asteroids, compared to the Bode-Titius predictions, in astronomical units, are as follows:

\begin{tabular}{|cccccccccc|}
\hline & Merc & Venus & Earth & Mars & Ceres & Jup & Sat & Uran & Nep \\
Obs: & 0.39 & 0.72 & 1.0 & 1.52 & 2.9 & 5.20 & 9.54 & 19.2 & 30.1 \\
B-T: & 0.4 & 0.7 & 1.0 & 1.6 & 2.8 & 5.2 & 10.0 & 19.6 & 38.8 \\
\hline
\end{tabular}

All planets from Mercury to Neptune revolve around the sun in the same direction. They move in elliptic orbits that depart only slightly from circles. All orbits are within $7^{\circ}$ of the ecliptic, defined by the earth's orbit. The sun and most of the planets rotate about their own axes in the same sense as the planetary revolution. The exceptions are Uranus, with an axis nearly in the plane of the ecliptic and Venus, in slow retrograde rotation.

Only a few of the more than fifty planetary satellites, including the large moon Triton around Neptune, are on retrograde orbits, either having been captured subsequent to the formation of the solar system, or having suffered some collision after formation. Despite these irregularities and the erratic motion of many comets and meteorites, the orderly behaviour of the major objects in the solar system could not very likely have resulted from chance encounters between hundreds of independent objects. The most telling argu- 
ment in favour of an orderly evolution of the solar system is still the BodeTitius law, which however, rarely receives serious consideration in modern cosmogony. As an important link in the self-similar chain from the infinitesimally small to the astronomically large, it certainly merits closer study.

To see if the orbital distances of the planets from the sun are commensurable in terms of simple rational fractions, the average distance of the remotest planet, Neptune, at 30AU is equated to unity. The other planetary distances, on the same scale are tabulated below. The average mean distance of the asteroids is $2.9 \mathrm{AU}$.

\begin{tabular}{|cccccccccc|}
\hline Sol & Merc & $\mathrm{V}$ & $\mathrm{E}$ & $\mathrm{M}$ & $\mathrm{C}$ & $\mathrm{J}$ & $\mathrm{S}$ & $\mathrm{U}$ & $\mathrm{N}$ \\
\hline$\frac{0}{1}$ & $\frac{1}{80}$ & $\frac{1}{40}$ & $\frac{1}{30}$ & $\frac{1}{20}$ & $\frac{1}{10}$ & $\frac{1}{6}$ & $\frac{1}{3}$ & $\frac{2}{3}$ & $\frac{1}{1}$ \\
$\mathrm{~d}$ & 0.38 & 0.75 & 1.0 & 1.5 & 3.0 & 5.0 & 10.0 & 20.0 & 30.0 \\
Obs & 0.39 & 0.72 & 1.0 & 1.52 & 2.9 & 5.2 & 9.5 & 19.2 & 30.3 \\
\hline
\end{tabular}

The distances described by the rational fractions are a considerable improvement over the Bode-Titius law. The overall sequence separates into two subsets at Ceres - the asteroid belt. This division corresponds to the division into four rocky planets of the inner solar system and four gaseous planets of the outer solar system, separated by the minor planets.

\begin{tabular}{|cccccc|c|ccccc|}
\hline Sol & Merc & V & E & M & C & & C & J & S & U & N \\
\hline$\frac{0}{1}$ & $\frac{1}{8}$ & $\frac{1}{4}$ & $\frac{1}{3}$ & $\frac{1}{2}$ & $\frac{1}{1}$ & & $\frac{1}{10}$ & $\frac{1}{6}$ & $\frac{1}{3}$ & $\frac{2}{3}$ & $\frac{1}{1}$ \\
\hline
\end{tabular}

The orbits from Venus to Ceres are represented by the Farey sequence $\mathcal{F}_{4}$, i.e. a unimodular set. The outer series is embedded in the sequence:

$$
\frac{1}{10}\left(\frac{1}{8} \frac{1}{7}\right) \frac{1}{6}\left(\frac{1}{5} \frac{1}{4}\right) \frac{1}{3}\left(\frac{3}{8} \frac{1}{2} \frac{4}{7}\right) \frac{2}{3}\left(\frac{5}{6}\right) \frac{1}{1}
$$

which is generated by a golden spiral with divergence angle of $\pi / 5\left(36^{\circ}\right)$, shown in Fig. 2. Numbers $(n)$ along the spiral correspond to angular displacements of $\theta=n \pi / 5$. The proportional lengths of successive distances from the spiral centre define the sequence (1) in reverse order. Assuming a divergence angle of $3 \pi / 5=108^{\circ}$ for the planetary spiral, shown in Fig. 3, only Uranus is out of place on the basis of predicted distances from the sun, at the centre of the spiral. 


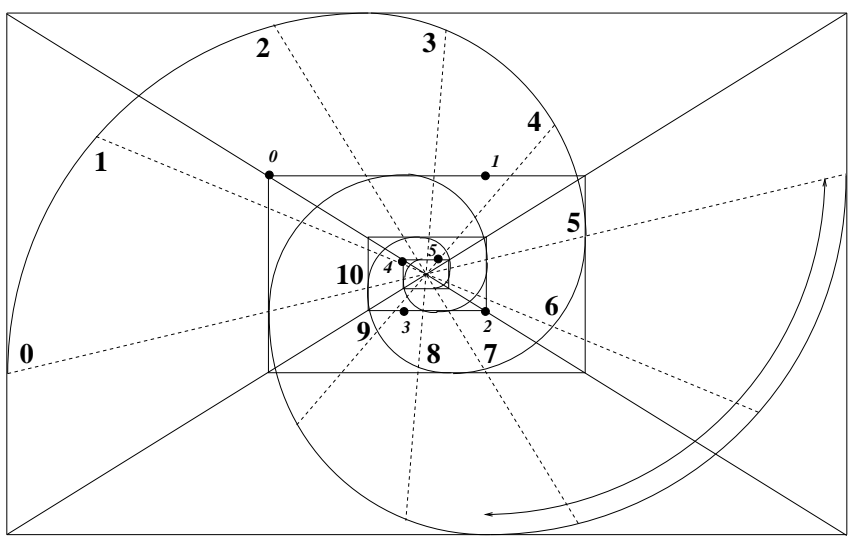

FIG. 2. A pair of logarithmic spirals inscribed in a golden rectangle. The reactangles of decreasing size are obtained by dividing the half-diagonals of a bigger rectangle in the golden ratio. Each spiral consists of circular segments centred at the points marked by small numerals. The first segment is marked on the spiral at the right.

The anomalous orientation of the rotation axis of Uranus has been ascribed ${ }^{3}$ to some collisional interaction, which could also have knocked the planet into the higher allowed orbit at $\theta=2 \pi / 5$.

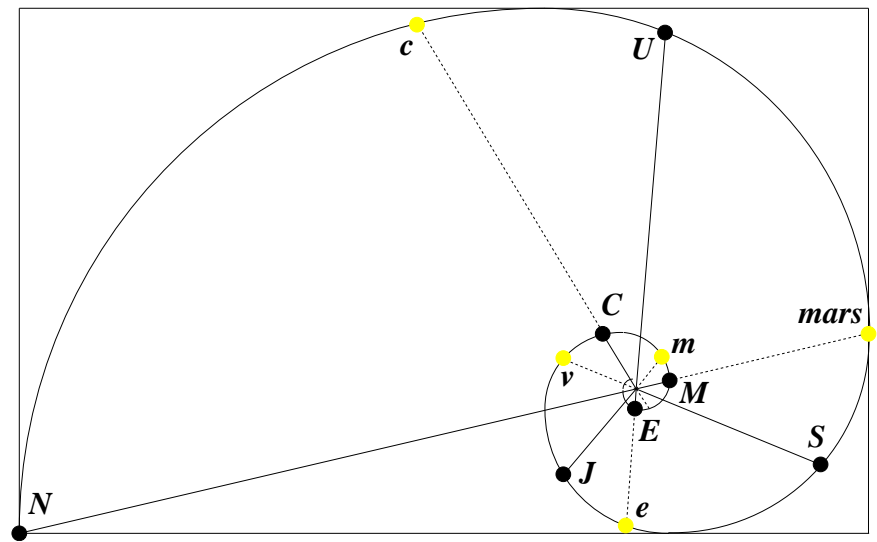

FIG. 3. Golden spiral with planetary positions as predicted by a divergence angle of $108^{\circ}$, starting from Neptune at the remote corner of a golden rectangle. Positions indicated by grey circles and marked in lower case refer to Ceres and the inner planets on a larger scale, consistent with the reduced scale that generates the black circles. 
The sequence is interrupted by the asteroid belt, made up of a myriad of minor planets around the expected position of a next planet. The sequence of inner planets continues with a divergence angle of $108^{\circ}$, starting from the middle of the asteroid belt.

One interpretation of this regular pattern is that the early solar system had a structure self-similar to that of a spiral galaxy, such as M51. To rationalize the appearance of elliptic orbits out of the double spiral, an inverse construction is shown in Fig. 4. Starting with a set of regularly spaced
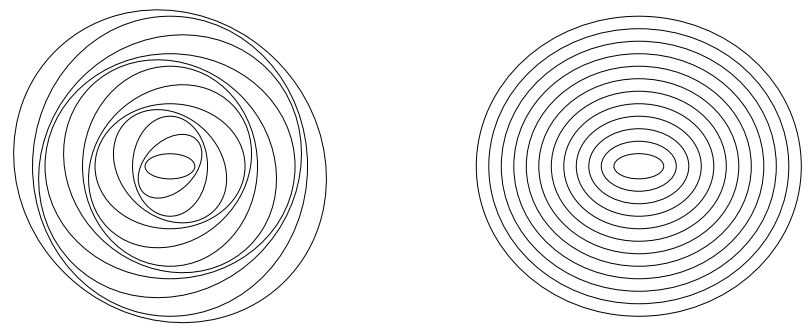

FIG. 4. Virtual spirals obtained by relative rotation of regular ellipses.

concentric ellipses each one is rotated through an angle of $45^{\circ}$ with respect to the previous member of the set. The resulting picture on the left shows two virtual spirals as in Fig. 1 and 2. Assuming the spiral structure to be generated by accelerated rotation, it follows that elliptic orbits should appear as rotation slows down.

\section{A. Kirkwood Gaps}

The Kirkwood gaps in the asteroid belt have been redetermined many times from histograms including more and more minor-planet coordinates. The gaps with periods of $\frac{3}{4}, \frac{1}{2}, \frac{3}{7}, \frac{1}{3}$ and $\frac{1}{4}$ of Jupiter's synodic year are the most prominent and those quoted by most authors. Expressed as fractions of Jupiter's mean distance from the sun, these periods correspond to the radial fractions $\frac{5}{6}, \frac{2}{3}, \frac{4}{7}, \frac{1}{2}, \frac{3}{8}$. These fractions coincide with the entries $n=1$ to 5 in Table 1 , but they refer to the orbit of Jupiter, not Neptune, as unity. Converted to the Neptune scale the Kirkwood gaps occur at fractional distances $\frac{1}{7}, \frac{1}{9}, \frac{1}{10}, \frac{1}{12}, \frac{1}{16}$. Accumulation of minor planets occur between the gaps and, referred to the spiral of Fig. 2, they occur, like the gaps with a divergence angle of $\pi / 5$. The minor planets, close to, or on the same orbit as Jupiter are known as the Trojan groups. A simplified histogram of the minor-planet distribution is shown in Fig. 5. Fractional radial coordinates 
are given for gaps, local minima and maxima in the asteroid count, scaled to Jupiter's orbit. Fractions in parentheses refer to the Neptune scale.

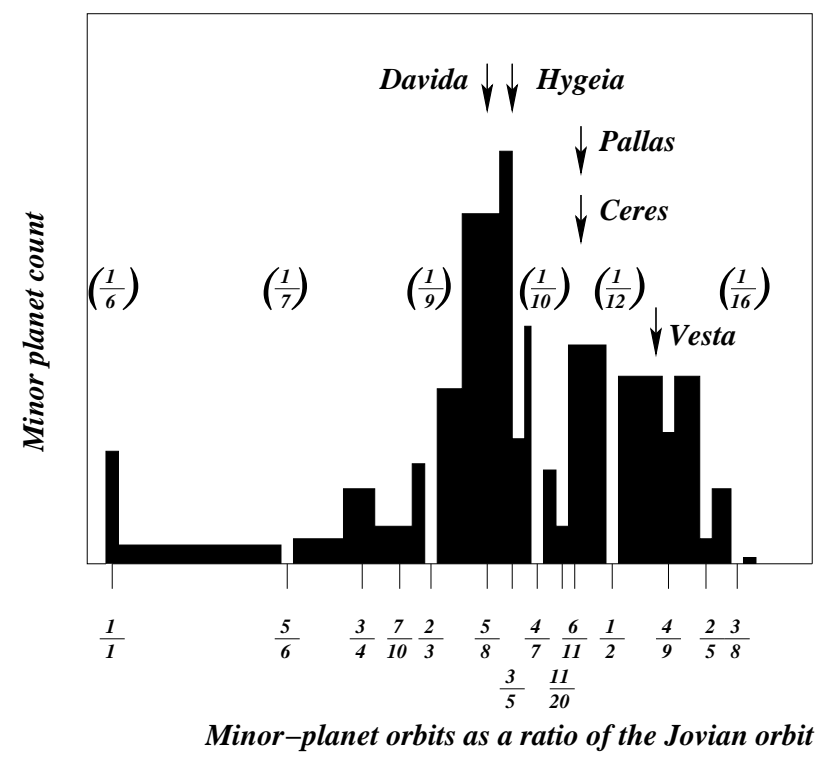

FIG. 5. Histogram of asteroid density showing Kirkwood gaps. Fractions in parentheses refer to the Neptune scale.

Arrows indicate the positions of the five largest of the minor planets: Ceres, Pallas, Vesta, Hygeia and Davida.

The density distribution in the asteroid belt follows a pattern like the spacing of planets around the sun, albeit on a different scale. The two patterns are said to be self-similar. All planets in the early solar system arguably consisted of fragmented building blocks on closely spaced orbits, as in the asteroid belt.

\section{PLANETARY SATELLITES}

The inferred divergence angle of $36^{\circ}$ suggests a basis for the arrangement of the moons that circle the major planets.

\section{A. Saturn}

Saturn, with eighteen, has the largest number of known moons. Two 
of these, Iapetus and Phoebe, are so remote from the planet that they are generally considered ${ }^{1}$ not to "date from the origin of the Saturnian system, but may have been captured by the planet at a later epoch". Nine other moons appear as groups on a common orbit, i.e. Epimetheus and Janus; Tethys, Telesto and Calypso; Dione and Helene; Pandora and Prometheus ${ }^{4}$. The expected commensurable relationship between the unique orbits is recognized directly by putting the orbital distance of Hyperion equal to one at $n=0$, as shown in Table 1 .

The largest discrepancy, of less than $5 \%$, occurs for the moon Rhea at $n=5$. The minor moon Janus at $n=12$ has the same relationship with Saturn as the minor planet Ceres has in the solar system. The four remaining moons are described ${ }^{5}$ as shepherding the ring system, which is centred at $n=12$. Pandora and Prometheus flank the F-ring. These satellites and the ring system occupy a region around Saturn equivalent to the asteroid belt in the solar system.

\section{B. Jupiter}

The many moons of Jupiter separate into three groups: the four Galilean moons, four minor moons on the inside and eight small moons, on highly inclined orbits, orders of magnitude more distant than the Galilean group. This latter group, like the outer moons of Saturn, have probably strayed into Jupiter's ambit from the asteroid belt. The Trojan group of asteroids are, for instance, on the same orbit as Jupiter. The Galilean satellites occur on $n \pi / 5$ orbits with Callisto at $n=0$ and most of the minor moons cluster around $n=10$, equivalent to the asteroid belt ${ }^{4}$. A ring system occurs inside the orbit of Metis and Adastrea.

\section{Uranus}

The moons of Uranus occur in commensurable sequence by placing Titania at $n=1$. This way the predicted position of Oberon deviates from the observed $^{4}$ by $10 \%$, while the others correspond exactly, as shown in Table 1. Six minor moons are clustered around the asteroid equivalent $(n=12)$ outside of a ring system centred at $n=15$. 
Table 1: Outer planets and moons

\begin{tabular}{|c|c|c|c|c|c|c|c|c|}
\hline $\mathrm{n}$ & & Sol & Uranus & Saturn & Jupiter & Mars & Neptune & $\mathrm{Q}$ \\
\hline 0 & $\begin{array}{l}\frac{1}{1} \\
5\end{array}$ & Neptune & $\begin{array}{l}\text { Oberon } \\
(+10 \%)\end{array}$ & Hyperion & Callisto & Deimos & & 6 \\
\hline 1 & $\frac{5}{6}$ & & Titania & Titan & & & & 5 \\
\hline 2 & $\frac{2}{3}$ & Uranus & & & & & & 4 \\
\hline 3 & $\frac{4}{7}$ & & & & Ganymede & & & \\
\hline 4 & $\frac{1}{2}$ & & Umbriel & & & & Triton & 3 \\
\hline 5 & $\frac{3}{8}$ & & & $\begin{array}{l}\text { Rhea } \\
(-5 \%)\end{array}$ & Europa & Phobos & & \\
\hline 6 & $\frac{1}{3}$ & Saturn & Ariel & & & & & 2 \\
\hline 7 & $\frac{1}{4}$ & & Miranda & Dione & & & & \\
\hline 8 & $\frac{1}{5}$ & & & Tethys & Io & & & \\
\hline 9 & $\frac{1}{6}$ & Jupiter & Puck & E-ring & & & Proteus & 1 \\
\hline 10 & $\frac{1}{7}$ & & Belinda & Enceladus & & & & \\
\hline 11 & $\frac{1}{8}$ & & Portia & Mimas & & & & \\
\hline 12 & $\frac{1}{10}$ & $\begin{array}{l}\text { Minor } \\
\text { Planets } \\
\text { Ceres } \\
\text { Minor } \\
\text { Planets }\end{array}$ & $\begin{array}{l}\text { Juliet } \\
\text { Desdemona } \\
\text { Cressida } \\
\text { Ophelia } \\
\text { Bianca } \\
\text { Cordelia }\end{array}$ & $\begin{array}{l}\text { Janus } \\
\text { Pandora } \\
\text { Prometeus } \\
\text { Atlas } \\
\text { Pan }\end{array}$ & $\begin{array}{l}\text { Amalthea } \\
\text { Adastrea }\end{array}$ & & $\begin{array}{l}\text { Larissa } \\
\text { Galatea } \\
\text { Despina } \\
\text { Thalassa } \\
\text { Naiad }\end{array}$ & $\tau$ \\
\hline
\end{tabular}

\section{Neptune}

Triton, the major satellite in retrograde rotation about Neptune, although probably captured from elsewhere, defines a commensurable sequence with the minor inner moons ${ }^{4}$. The moon Nereid with its highly eccentric orbit, an order of magnitude further away than Triton is excluded from consideration. Placing Triton at $n=4$, Larissa $(n=12)$ is the asteroid 
equivalent, with a smaller cluster shepherding a complicated ring system.

\section{E. Mars}

The two moons of Mars placed at $n=0$ (Deimos) and $n=5$ (Phobos) obey the rule that applies to the moons of the outer planets.

\section{F. The Rings of Saturn}

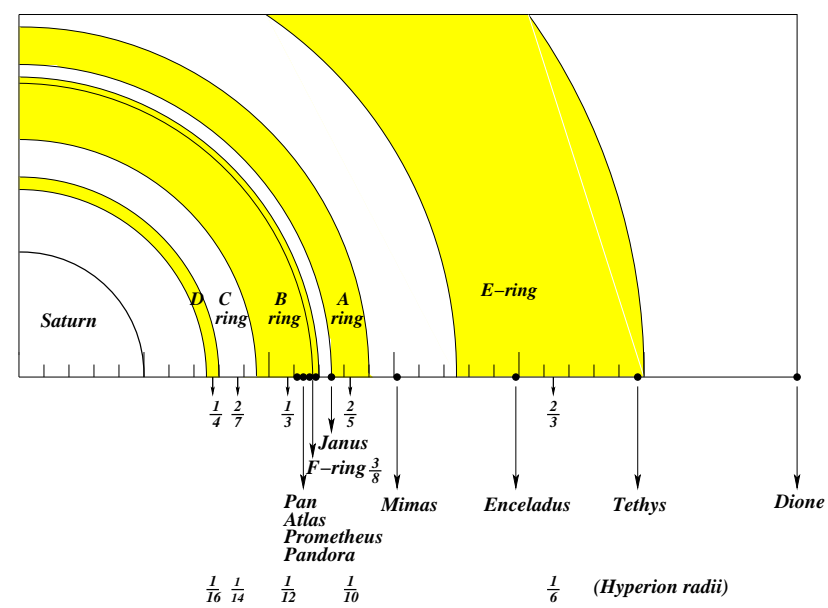

FIG. 6. (Color online) The rings and minor moons of Saturn.

Saturn has the most extensive and complex ring system in the solar system. The information radioed back by the Voyager 2 spacecraft indicates a system consisting of thousands of rings, but these can be grouped into four or five clusters. Best known and clearly visible from earth are the A and $\mathrm{B}$ rings, separated by the Cassini division, which resembles the Kirkwood gaps. On the inside of the B-ring is the more diffuse $\mathrm{C}$ and $\mathrm{D}$ rings. The inconspicuous F-ring is flanked by several minor moons. The large E-ring lies much further out.

The orbits of minor moons and edges of the major ring systems are shown on scale in Fig. 6. All radii are in units of Saturn's radius of $60330 \mathrm{~km}$. Fractional radii are with respect to the orbit of Dione. With Hyperion as reference these factors are to be divided by 4 . The average radii of the rings E, A, B, C, D are readily fitted to the fractions $\frac{2}{3}, \frac{2}{5}, \frac{1}{3}, \frac{2}{7}$, and $\frac{1}{4}$ of Dione. 


\section{DISCUSSION}

The symmetry, which is noticed on comparison of Table 1 with Fig. $1-3$, suggests that each planetary system is like a miniature solar system and each solar system is like a miniature spiral galaxy ${ }^{1}$. It is especially noticeable how both planets and moons tend to occur as antipodal pairs: Neptune-Mars, (Uranus)-Earth, Saturn-Venus and Jupiter-Mercury among planets; and Oberon-Ariel-Belinda, Hyperion-Rhea-Enceladus, CallistoEuropa, Deimos -Phobos, Ganymede-Io, Triton-Proteus, Dione-Janus, Umbriel-Puck, among the moons.

All of the major planets have ring systems with shepherding satellites that correspond to the solar asteroid belt. The gaps in both the asteroid belt and planetary rings are commensurable with a common algorithm.

One inference to be drawn from the appearance of self-similar astronomical structures is that in all cases the structures develop by a common mechanism, such as rotation about a central point in a nebular cloud. It would not be too surprising if the set of elliptical orbits generated in the process exhibited similar geometrical patterns. It is more surprising however, if a foreign body such as Triton, when captured into a stable planetary system, selects an orbit that fits the evolutionary geometrical pattern. It is equally surprising to find the planet Uranus, being knocked from its natural orbit, not into some random orbit, but again into one that fits the pattern. For both planets and moons there seems to exist some specific allowed orbits as in the Bohr-Sommerfeld model of an atom. It is almost predictable that the Aufbau procedure that underpins the periodic table of the elements is based, as found, on the same numerical structure of a golden rectangle ${ }^{2}$.

The symmetrical separation of outer and inner planets by an asteroid belt is not accidental and merits further analysis. An equivalent separation between major moons and planetary rings by clusters of lesser moons occurs for the four outer planets. The last column of Table 1 shows that the orbits of all outer planets are specified by integers on choosing the Jovian orbit as unity. The mean orbit of the asteroid belt, on the same scale, is then given by the golden ratio $\tau=0.618034 \ldots$ and those of the inner planets by $\tau / n, n>1$. These orbital integers, as shown in Fig. 7 , operate like quantum numbers for the planets, but not for minor planets or planetary satellites. However, the radial scale for some planetary satellites was fixed by




an arbitrary choice of unit radius for the outermost major moon. If, instead these moons are placed at a radius of $\frac{5}{6}$ most of the major moons also have integer $Q$-numbers, as shown in an updated Table 2 . The only exceptions are Miranda and Dione, for which there is a plausible rationalization.

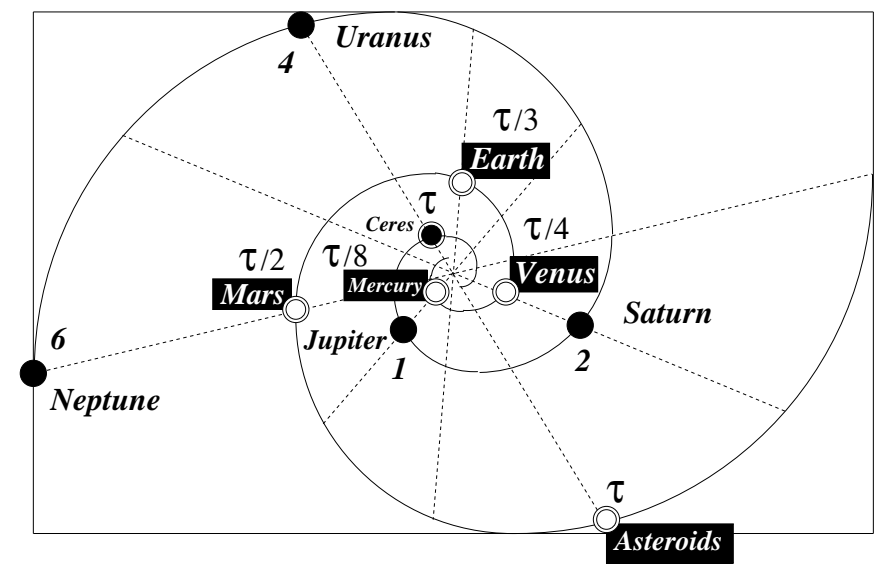

FIG. 7. Planetary orbits defined as products of $Q$-numbers and the mean Jovian orbit. Orbits of the inner planets are shown on a larger, self-similar, scale.

The asteroid belt between Mars and Jupiter is approximately 1.5AU wide. Each of the Trojan groups of asteroids forms an equilateral triangle with the sun and Jupiter and there are thousands of minor planets scattered on the same orbit as Jupiter, or close to it. Disturbances from the chaotic region centred at $Q=\tau$ therefore extend well beyond the Jovian orbit. A comparable situation is likely in the Uranian and Saturnian systems.

The tiny moon, Miranda, at $5 \%$ the mass of neighbouring Ariel $^{4}$, and judging by its battered appearance ${ }^{5}$, is clearly such an invader from the chaotic zone. In the Saturnian system the moon, Dione, although considerable larger, but moving in tandem with the smaller Helene and companions Telesto and Calypso, on the same orbit as Tethys, must also be considered an invader.

The recalculated whole-number rule is postulated to operate in all dynamically stable arrangements in the solar system and has little effect on the description of the chaotic region of rings and minor moons. It does remove Saturn's E-ring from the sphere of major moons. As the asteroid belt starts at the Jovian orbit, so the Saturnian ring system now also starts at the equivalent point (orbit of Tethys) in the Saturnian system. 
Table 2: Recalculated positions of major moons

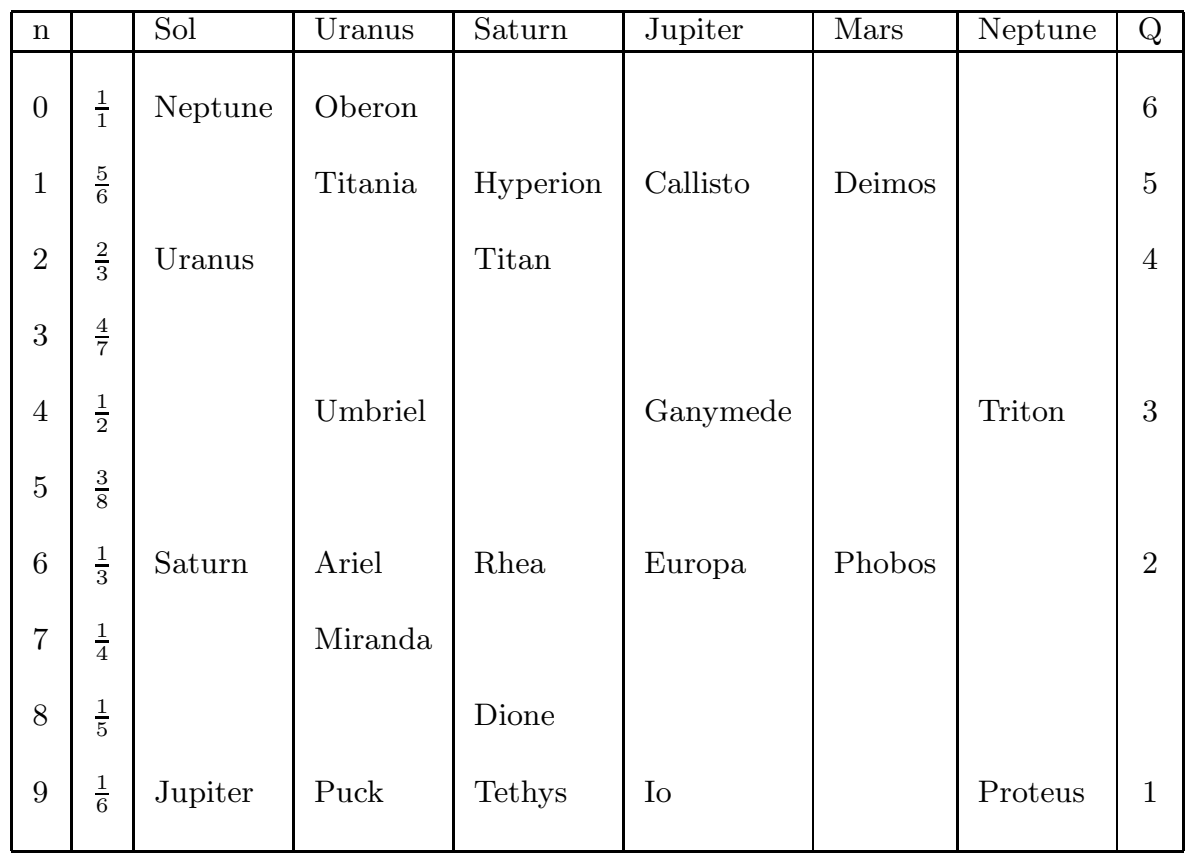

The $Q$-pattern suggests a provocative alternative explanation for the anomalous orbit of Uranus. Only by breaking the constant divergence-angle rule can the planet find an orbit with integer $Q$, which may well be the stronger criterion. Jupiter emerges as the dominant factor, next to the sun, in shaping the solar system. Other solar systems, which have been observed, all seem to be dominated by a single giant planet. Improved resolution could conceivably reveal secondary structure of the type described here. The irrational $Q$-number for the asteroid belt provides an intriguing clue to the chaotic situation that prevails in this region and the number equivalent of the break between outer and inner planets makes intuitive sense.

The common botanical divergence angle is $2 \pi \tau^{2}$ radians $\left(137.5^{\circ}\right)$, compared to $(3 / 5) \pi \simeq \pi \tau\left(108^{\circ}\right)$ of the planetary spiral. The botanical angle allows maximum radiation to reach each leaf and is an area effect. Competition for intermediate material, along a semi-circle in the spiral arm, would generate a divergence angle with linear dependence on $\tau$. It was suggested ${ }^{6}$ that the divergence angle may be a consequence of optimized accretion of material in the original cloud, noting that optimization problems governed by second-order dynamics can be solved by logarithmic spirals. 
The phase trajectory for two-dimensional complex eigenvectors $\left(\lambda_{1,2}=\right.$ $a+i b, b \neq 0$ ), in polar coordinates,

$$
\rho=K e^{(a / b) \varphi}
$$

defines a logarithmic spiral ${ }^{7}$ (p. 144) for $(a \neq 0)$. Different phase trajectories are related by dilation, centred at the origin - the property invoked in Fig. 7. It is inferred that our $Q$-numbers define eigenvalues for the optimization of planet formation in the planar ecliptic, under central-field gravitation as control parameter. As in the Bohr model of the atom, allowed orbits are selected by angular-momentum eigenvalues, noting that orbital angular momentum is quantum-mechanically also specified by complex eigenvectors.

On defining dimensionless astronomical units, by setting the mass, orbital period and semi-major axis $(a)$ of planet Earth equal to unity, Kepler's constant, $r^{3} / t^{2}=v^{2} r$, reduces to unity and the squared angular momentum per unit mass, for any planet, $(L / m)^{2}=(v r)^{2}$ is numerically equal to $r$. For the outer planets with $Q$-number $l,(L / m)^{2}=l H$, and for the inner planets $(L / m)^{2}=\tau H / l$, with $H=4.9 \mathrm{AU}$. In the Bohr model, which refers to three-dimensional rotation, $L_{z}=m_{l} \hbar$. Some results are summarized in Table 3 , in units of $\mathrm{km} / 10^{6}$ and in dimensionless AU.

Table 3: Planetary data

\begin{tabular}{|c|c|c|c|c|c|c|c|c|c|c|c|}
\hline Planet & $l$ & Mass & \multicolumn{2}{|c|}{$\mathrm{a}(l \mathrm{H})$} & \multicolumn{2}{c|}{$\mathrm{a}($ Observed $)$} & Error & $v$ & $L / m$ & $v r$ & $L$ \\
\cline { 4 - 9 } Mercury & 8 & 0.0553 & 56.7 & 0.38 & 58.0 & 0.39 & -2 & 1.607 & 0.62 & 0.63 & 0.03 \\
Venus & 4 & 0.8150 & 113.4 & 0.76 & 108.1 & 0.72 & +5 & 1.176 & 0.87 & 0.85 & 0.71 \\
Earth & 3 & 1.000 & 151.2 & 1.00 & 149.6 & 1.00 & +1 & 1.000 & 1.00 & 1.00 & 1.00 \\
Mars & 2 & 0.1074 & 226.8 & 1.51 & 227.8 & 1.52 & 0 & 0.8102 & 1.23 & 1.23 & 0.13 \\
Minors & 1 & & 453.6 & 3.0 & 435 & 2.9 & +4 & & & & \\
Jupiter & 1 & 317.8 & 734 & 4.9 & 778 & 5.2 & -5 & 0.4384 & 2.22 & 2.28 & 705 \\
Saturn & 2 & 95.16 & 1468 & 9.8 & 1427 & 9.5 & +3 & 0.3237 & 3.13 & 3.08 & 298 \\
Uranus & 4 & 14.50 & 2936 & 19.6 & 2870 & 19.2 & +2 & 0.2282 & 4.44 & 4.38 & 64 \\
Neptune & 6 & 17.20 & 4404 & 29.4 & 4500 & 30.3 & -2 & 0.1823 & 5.42 & 5.52 & 93 \\
Pluto & 8 & 0.01 & 5872 & 39.1 & 5909 & 39.4 & -1 & 0.1591 & 6.27 & 6.27 & 0.06 \\
\hline
\end{tabular}


These results support the conjecture ${ }^{8}$ that the universe is self-similar from the subatomic to the astronomical scale, but not the inference that the universe has a memory of some quantum origin. The $Q$-numbers do not appear in conjunction with Planck's constant.

Any system of planets and moons in the solar system may now be considered made up of a nucleus and a commensurable core, consisting of three regions. The fully commensurable outer and inner regions are separated by a more chaotic central band, in partial overlap with the inner and outer regions. Overlap with an inner region is recognized in the regular appearance of meteorites and the Apollo asteroids on near-Earth orbits ${ }^{9,10}$.

Pluto, with its companion Charon, and the distant moons of Jupiter, Saturn and Neptune are not part of such a core. Also on the outside are comets, and for lack of better terminology, planetoids of uncertain origin. The object Chiron, once considered to be an asteroid, is now classified as a comet with a coma. Long-period comets are assumed to originate in the Oort cloud and short-period comets, including Halley, in the Kuiper belt, on the ecliptic, outside Neptune's orbit ${ }^{11}$. Whatever the nature of these objects they are inevitably remnants of the nebular cloud from which the solar system condensed in the first place. First-neighbour stars in the Milky Way are considered too remote to interact directly with the solar system by way of interstellar comets.

The commensurable pattern which is recognized here holds the promise of better understanding of the structure, origin and evolution, not only of the solar system, but also of all the different types of galaxy. It argues convincingly against a unique solar system in the cosmos.

\section{ACKNOWLEDGEMENTS}

The important advice of Max Braun, Demi Levendis and Johan Malherbe is gratefully acknowledged.

${ }^{1}$ G.E. Satterthwaite, Encyclopedia of Astronomy, Hamlyn, London, (1970).

2 J.C.A. Boeyens, Chemistry from First Principles, Springer.com., (2008).

${ }^{3}$ F. Hoyle, Frontiers of Astronomy, Heineman, London, (1955).

${ }^{4}$ D.R. Lide (ed.) CRC Handbook of Chemistry and Physics, 86th ed., Taylor and Francis, Boca Raton, (2005).

${ }^{5}$ E.C. Stone, The Journeys of the Voyagers, Encyclopædia Britannica, Science and the Future, 27-41, (1991).

${ }^{6}$ M.W.H. Braun, Personal communication, (2008).

${ }^{7}$ V.G. Boltyanskii, Mathematical Methods of Optimal Control, translated 
from the Russian by K.N. Trirogoff and I. Tarnove, Holt, Rinehart and Winston, NY, (1971).

${ }^{8}$ G. Iovane, E. Laserra, F.S. Tortoriello, Chaos, Solitons and Fractals, 20, 425-426, (2004).

${ }^{9}$ I. Peterson, Newton's Clock, Freeman, NY, (1993).

${ }^{10}$ C.R. Chapman, Asteroids: Secrets Locked in Stone, Encyclopædia Britannica, Science and the Future, 76-99, (1996).

${ }^{11}$ R.A. Kerr, Science, 239, 1372, (1998). 